\title{
Quantum Mechanical Models for Organometallic Reactivity
}

\author{
A. L. TCHOUGRÉEFF \\ L. Y. Karpov Institute of Physical Chemistry, Vorontsovo pole 10, 103064 Moscow, Russia
}

Received April 30, 1993; revised manuscript received February 15, 1995; accepted June 12, 1995

\begin{abstract}
A general scheme for theoretical treatment of organometallic reactivity is proposed. It is based upon the notion that the reactivity of a molecule is strongly affected by its coordination to metal-containing fragments. Based upon this idea we describe the large-scale organometallic reactions as reactions of the ligands in the coordination spheres of transition metal complexes. We propose here a quantum mechanical framework for analysis of effects of coordination on the reactivity and give several examples of qualitative energy profiles for reactions in the ligand spheres of transition metal complexes. (c) 1996 John Wiley \& Sons, Inc.
\end{abstract}

\section{Introduction}

$\mathbf{T}$ he problem of organometallic reactivity is important in view of its applications to organic and organometallic synthesis, preparative and industrial catalysis, biochemistry, etc. (for review see $[1-4])$. For this reason an attempt to analyze this problem in terms of quantum mechanics is of interest. Theoretical work in this area is largely presented by state-of-the-art calculations on reaction paths and activation energies of various processes of particular importance (see, e.g., reviews [5] and a recent systematic calculation [6]). Our purpose is, however, to consider a more general perspective of the problem.
An important fact to be taken into account while constructing a general approach to the organometallic reactivity is the local character of chemical interactions. If the molecule is large enough (and organometallic molecules usually are), chemical transformations touch only a relatively small part of the whole, whereas other parts of the molecule remain unchanged. Although this simple fact is obviously the basis for all the synthetic organic and organometallic chemistry, it is by no means reflected in the quantum methods used for calculations of the reaction paths of organometallic reactions [7]. The calculation methods use the molecular orbitals (MO) taken as linear combinations of atomic orbital (LCAO) $[8,9]$, and the problem originate from the LCAO approximation. It describes the electronic structure in terms 
of the MO-LCAO. These canonical MOs are spread over whole molecule, and this forces students to deal with the entire, complex molecule rather than with its interesting reactive part.

It is natural to divide the reacting organometallic (super)molecule into (i) the small part to which the chemical transformation actually happens, the reactive subsystem (RS), and (ii) the remainder of the supermolecule. In this article we will discuss only those organometallic reactions in which the reactive subsystem is purely organic and does not contain the transition metal atom. We call the intact remainder of the supermolecule-the metal-containing fragment-the catalyst subsystem (cs) regardless of whether the reaction is catalytic in the strict sense.

Approaches based on decomposition of systems were developed in the area of surface chemistry to describe the reactivity of adsorbed molecules [10-14]. The approach proposed in [14] seems to be particularly attractive because it attacks the problem of surface reactivity directly. It starts from the potential energy surfaces (PESs) of the free reagents in the gas phase. The data on their absorption energies are used to parameterize interactions of the reagents with the surface, and the bond order conservation principle is then applied to interpolate the energy profile of the surface reaction. However, the method [14] is not a quantum mechanical one. We have no information about the details of the electronic structure of the surface and of reacting molecules, which are responsible for the modification of the energy profile under absorption.

From the general quantum mechanical point of view the presentation of a system as a composition of smaller parts implies that the states of the system are to be expressed in terms of the states of the constituent subsystems. In quantum chemistry this idea was introduced by Moffitt [15] in his method of "atoms in the molecules" or, more generally, "molecules in the molecules" (see also [16]). In the framework of this approach the electronic state of a molecule is presented as a composition of the states of the constituent atoms (or more generally smaller molecules). Interactions between the atoms modify their states, but the experimental information is available on many atomic states and we can use it to describe properties of the molecule. In our case of organometallic reactivity the states of the organometallic supermolecule must be expressed in terms of the states of the reactive and catalyst subsystems (RS and CS).

\section{Theory}

\section{GENERAL THEORY FOR PES}

According to the general quantum chemical approach to the problem of chemical reactivity, the PES $E(q)$ for any reaction in the ground state is the function of the full set of the nuclear coordinates $q$ (see, e.g., [9]):

$$
E(q)=\left\langle\Psi^{0}(q)|H(q)| \Psi^{0}(q)\right\rangle,
$$

where $\Psi^{0}(q)$ is the electronic ground-state wave function for the nuclear configuration $q$ and $H(q)$ is the electron Hamiltonian for the system, also depending on the nuclear configuration.

Let $\Phi_{R}^{i}(q)$ and $\Phi_{M}^{k}(q)$ be the orthonormal many-electron wave functions (may be approximate) for the $i$ th eigenstate of the electrons in the RS and for the $k$ th eigenstate of the electrons in the $\mathrm{CS}$, respectively. The ground-state electronic wave function of the complex can be presented in the form [17]

$$
\begin{gathered}
\Psi^{0}(q)=\sum_{i, k} C_{i k}^{0}(q) \Phi_{R}^{i}(q) \wedge \Phi_{M}^{k}(q) \\
\sum_{i, k} C_{i k}^{0}(q)^{2}=1, \quad i, k=0,1, \ldots
\end{gathered}
$$

where $C_{i k}^{0}(q)$ are the numerical coefficients and the symbol $\wedge$ stands for the antisymmetrized product. It ensures the antisymmetry of the orthonormalized product functions with respect to permutations of electron coordinates.

The Hamiltonian of the whole system can also be presented as the sum

$$
H(q)=H_{R}(q)+H_{M}(q)+H_{i}(q),
$$

where $H_{R}(q)$ is the Hamiltonian for the electrons in the RS, $H_{M}(q)$ is the Hamiltonian for the electrons in the Cs, and $H_{i}(q)$ is the operator describing all the interactions between the two subsystems.

The RS of the supermolecule is that part in which the new bonds are formed and the old ones are cleaved. The major changes of the nuclear configuration occur also only in the RS, whereas the nuclear coordinates of the CS remain nearly constant. (We can, of course, redefine the RS to include some atoms in it if their relative positions 
vary significantly.) In that case we can restrict the set of nuclear coordinates $q$ to the nuclear coordinates of the RS only.

Now we consider the PES for the supermolecule. First, we set $H_{\text {int }}=0$. If the ground state of at least one of the subsystems is nondegenerate, the ground-state wave function of the supermolecule takes the simple form

$$
\Psi_{o}^{0}(q)=\Phi_{R}^{0}(q) \wedge \Phi_{M}^{0},
$$

where the wave functions $\Phi_{R}^{0}(q)$ and $\Phi_{M}^{0}$ are respectively the ground-state wave functions for the RS and CS. Since the interaction is vanishing, the nuclear motions in the RS do not affect the wave function of the CS $\Phi_{M}^{0}$. The PES of the supermolecule in that case coincides with that for the free Rs but is shifted by the constant energy of the ground state of the cs:

$$
\begin{aligned}
E(q)= & \left\langle\Psi_{o}^{0}(q)|H(q)| \Psi_{o}^{0}(q)\right\rangle \\
= & \left\langle\Phi_{R}^{0}(q) \wedge \Phi_{M}^{0}\left|H_{R}(q)+H_{M}\right| \Phi_{R}^{0}(q) \wedge \Phi_{M}^{0}\right\rangle \\
= & E_{R}^{0}(q)+E_{M}, \\
& E_{R}^{0}(q)=\left\langle\Phi_{R}^{0}(q)\left|H_{R}(q)\right| \Phi_{R}^{0}(q)\right\rangle, \\
& E_{M}^{0}=\left\langle\Phi_{M}^{0}\left|H_{M}\right| \Phi_{M}^{0}\right\rangle .
\end{aligned}
$$

When the interaction is turned on, the excited and/or ionized states of both the RS and CS are populated. Inserting Eqs. (2) and (3) into Eq. (1) and taking into account that the states $\Phi_{R}^{i}(q)$ and $\Phi_{M}^{k}$ are the eigenstates of the operators $H_{R}(q)$ and $H_{M}$ and thus only the operator $H_{i}(q)$ can mix the states with $i, k \neq i^{\prime}, k^{\prime}$, we obtain

$$
\begin{aligned}
E(q)= & \left\langle\Psi^{0}(q)|H(q)| \Psi^{0}(q)\right\rangle \\
= & \sum_{i, k} C_{i k}^{0}(q)^{2}\left\langle\Phi_{R}^{i}(q) \wedge \Phi_{M}^{k}\right| H_{R}(q) \\
& +H_{M}\left|\Phi_{R}^{i}(q) \wedge \Phi_{M}^{k}\right\rangle \\
& +\sum_{i, k} \sum_{i^{\prime}, k^{\prime}} C_{i k}^{0}(q) C_{i^{\prime} k^{\prime}}^{0}(q) \\
& \times\left\langle\Phi_{R}^{i}(q) \wedge \Phi_{M}^{k}\left|H_{i}(q)\right| \Phi_{R}^{i^{\prime}}(q) \wedge \Phi_{M}^{k^{\prime}}\right\rangle \\
= & \sum_{i, k} C_{i k}^{0}(q)^{2}\left(\left\langle\Phi_{R}^{i}(q)\left|H_{R}(q)\right| \Phi_{R}^{i}(q)\right\rangle\right. \\
& \left.+\left\langle\Phi_{M}^{k}\left|H_{M}\right| \Phi_{M}^{k}\right\rangle\right) \\
& +\sum_{i, k} \sum_{i^{\prime}, k^{\prime}} C_{i k}^{0}(q) C_{i^{\prime} k^{\prime}}^{0}(q) \\
& \times\left\langle\Phi_{R}^{i}(q) \wedge \Phi_{M}^{k}\left|H_{i}(q)\right| \Phi_{R}^{i^{\prime}}(q) \wedge \Phi_{M}^{k^{\prime}}\right\rangle
\end{aligned}
$$

$$
\begin{aligned}
= & \sum_{i, k} C_{i k}^{0}(q)^{2}\left(E_{R}^{i}(q)+E_{M}^{k}\right) \\
& +\sum_{i, k} \sum_{i^{\prime}, k^{\prime}} C_{i k}^{0}(q) C_{i^{\prime} k^{\prime}}^{0}(q) \\
& \times\left\langle\Phi_{R}^{i}(q) \wedge \Phi_{M}^{k}\left|H_{i}(q)\right| \Phi_{R}^{i^{\prime}}(q) \wedge \Phi_{M}^{k^{\prime}}\right\rangle .
\end{aligned}
$$

The expression for the PES of the supermolecule [Eq. (6)] contains two groups of terms. The first group is the double sum over the states of the subsystems. Each term in this sum is a PES of the free RS in the $i$ th excited or ionized state multiplied by the weight factors $C_{i k}^{0}(q)^{2}$. By this admixture the PES of the excited or ionized states contributes to that of the supermolecule in its ground state. Analysis of these contributions gives us important hints concerning the shape of the groundstate PES of a supermolecule and also provides a qualitative description of the PES in terms of those of the eigenstates of the RS. If the ground-state energy of the RS increases along a certain path in the nuclear configuration space, the energy of the supermolecule is likely to increase along that path. However, if the energies of some excited/ionized states of the RS, giving significant contribution to the ground state of the supermolecule, decrease along the same path, the increase of the supermolecular ground-state energy along this path will be less pronounced and even may change to decrease.

The effect of the second group of the terms (the fourfold sum over the subsystem states) is not so clear. These terms describe the bonding interaction between the reacting ligands and the catalyst and give a stabilizing contribution to the total energy of the supermolecule.

\section{TWO-LEVEL APPROXIMATION FOR PES}

Here we consider a simplified two-level model for the PES of the organometallic supermolecule. We assume that the ground-state wave function (2) of the supermolecule has the form

$$
\Psi^{0}(q)=\cos \varphi(q) \psi_{g}+\sin \varphi(q) \psi_{e},
$$

where $\varphi(q)$ is a variational parameter.

Let the ground state of the RS be a ${ }^{2 S_{R}+1} \Gamma_{R}$ term and that of the CS be a ${ }^{2 S_{M}+1} \Gamma_{M}$ term. (Here $\Gamma_{R}$ and $\Gamma_{M}$ are the irreducible representations of the point group of the supermolecule and $S_{R}$ and $S_{M}$ are the spin quantum numbers.) We assume that the supermolecule ground state is a ${ }^{2 S+1} \Gamma$ multiplet. Then we take its component with the spin projec- 
tion $\sigma$, belonging to the specific row $\gamma$ as the ground-state function of the supermolecule. The basis functions $\psi_{g}$ and $\psi_{e}$ are also the ${ }^{2 S+1} \Gamma$ multiplet states with the same $\sigma$ and $\gamma$.

The antisymmetrized product of the ground states of the RS and the CS probably gives the leading contribution to the ground state of the supermolecule. Thus the $\psi_{g}$ basis function is a symmetry-adapted antisymmetrized product of the ground-state multiplet functions of the RS and the CS:

$$
\begin{aligned}
\psi_{g}= & \sum_{\gamma_{M}, \gamma_{R}} \sum_{\sigma_{M}, \sigma_{R}}\left(\Gamma \gamma \mid \Gamma_{M} \gamma_{M}, \Gamma_{R} \gamma_{R}\right) \\
& \times\left(S \sigma \mid S_{M} \sigma_{M}, S_{R} \sigma_{R}\right) \\
& \times\left|\Gamma_{M} \gamma_{M} S_{M} \sigma_{M}\right\rangle \wedge\left|\Gamma_{R} \gamma_{R} S_{R} \sigma_{R}\right\rangle,
\end{aligned}
$$

where $\left(\Gamma \gamma \mid \Gamma_{M} \gamma_{M}, \Gamma_{R} \gamma_{R}\right)$ and $\left(S \sigma \mid S_{M} \sigma_{M}, S_{R} \sigma_{R}\right)$ are the vector coupling coefficients for the point group and for the spin, respectively [18].

The $\psi_{e}$ function is also the $(\sigma, \gamma)$ component of the ${ }^{2 S+1} \Gamma$ multiplet build of the ${ }^{2 S_{R}^{\prime}+1} \Gamma_{R}^{\prime}$ and ${ }^{2 S_{M}^{\prime}+1} \Gamma_{M}^{\prime}$ excited/ionized states of the respective subsystems. It has the form

$$
\begin{aligned}
\psi_{e}= & \sum_{\gamma_{M}, \gamma_{R}} \sum_{\sigma_{M}, \sigma_{R}}\left(\Gamma \gamma \mid \Gamma_{M}^{\prime} \gamma_{M}, \Gamma_{R}^{\prime} \gamma_{R}\right) \\
& \times\left(S \sigma \mid S_{M}^{\prime} \sigma_{M}, S_{R}^{\prime} \sigma_{R}\right) \\
& \times\left|\Gamma_{M}^{\prime} \gamma_{M} S_{M}^{\prime} \sigma_{M}\right\rangle \wedge\left|\Gamma_{R}^{\prime} \gamma_{R} S_{R}^{\prime} \sigma_{R}\right\rangle .
\end{aligned}
$$

The ground-state wave function $\Psi^{0}(q)$ of the supermolecule can be found for any point $q$ in the nuclear configurational space by the formulas.

$$
\begin{aligned}
& \tan 2 \varphi(q)=\frac{2 b}{\Delta E_{g e}}, \\
& b=\left\langle\psi_{g}\left|H_{i}(q)\right| \psi_{e}\right\rangle, \\
& \Delta E_{g e}=\left\langle\psi_{e}|H| \psi_{e}\right\rangle-\left\langle\psi_{g}|H| \psi_{g}\right\rangle, \\
&\left\langle\psi_{g}|H(q)| \psi_{g}\right\rangle=\left\langle\psi_{g}\left|H_{R}(q)+H_{M}+H_{i}(q)\right| \psi_{g}\right\rangle \\
&=\left\langle\Gamma_{R} S_{R}\left|H_{R}(q)\right| \Gamma_{R} S_{R}\right\rangle \\
&+\left\langle\Gamma_{M} S_{M}\left|H_{M}\right| \Gamma_{M} S_{M}\right\rangle \\
&+\left\langle\psi_{g}\left|H_{i}(q)\right| \psi_{g}\right\rangle, \\
&\left\langle\psi_{e}|H(q)| \psi_{e}\right\rangle=\left\langle\psi_{e}\left|H_{R}(q)+H_{M}+H_{i}(q)\right| \psi_{e}\right\rangle \\
&=\left\langle\Gamma_{R}^{\prime} S_{R}^{\prime}\left|H_{R}(q)\right| \Gamma_{R}^{\prime} S_{R}^{\prime}\right\rangle \\
&+\left\langle\Gamma_{M}^{\prime} S_{M}^{\prime}\left|H_{M}\right| \Gamma_{M}^{\prime} S_{M}^{\prime}\right\rangle \\
&+\left\langle\psi_{e}\left|H_{i}(q)\right| \psi_{e}\right\rangle .
\end{aligned}
$$

The PES in the two-level approximation also can be written immediately:

$$
\begin{aligned}
E(q)= & \left(E_{R}(q)+E_{M}+\left\langle\psi_{g}\left|H_{i}(q)\right| \psi_{g}\right\rangle\right) \cos ^{2} \varphi(q) \\
= & \left(E_{R}^{\prime}(q)+E_{M}^{\prime}+\left\langle\psi_{e}\left|H_{i}(q)\right| \psi_{e}\right\rangle\right) \sin ^{2} \varphi(q) \\
& +b \sin 2 \varphi(q)
\end{aligned}
$$

or

$$
\begin{aligned}
E(q)= & \left(E_{R}(q)+E_{M}+\left\langle\psi_{g}\left|H_{i}(q)\right| \psi_{g}\right\rangle\right) \\
& +\left(\Delta E_{R}(q)+\Delta E_{M}+\Delta E_{i}\right) \sin ^{2} \varphi(q) \\
& +b \sin 2 \varphi(q)
\end{aligned}
$$

where the obvious notation

$$
\begin{aligned}
E_{R}(q) & =\left\langle\Gamma_{R} S_{R}\left|H_{R}(q)\right| \Gamma_{R} S_{R}\right\rangle, \\
E_{R}^{\prime}(q) & =\left\langle\Gamma_{R}^{\prime} S_{R}^{\prime}\left|H_{R}(q)\right| \Gamma_{R}^{\prime} S_{R}^{\prime}\right\rangle, \\
E_{M} & =\left\langle\Gamma_{M} S_{M}\left|H_{M}\right| \Gamma_{M} S_{M}\right\rangle, \\
E_{M}^{\prime} & =\left\langle\Gamma_{M}^{\prime} S_{M}^{\prime}\left|H_{M}\right| \Gamma_{M}^{\prime} S_{M}^{\prime}\right\rangle, \\
\Delta E_{R}(q) & =E_{R}^{\prime}(q)-E_{R}(q), \quad \Delta E_{M}=E_{M}^{\prime}-E_{M}, \\
\Delta E_{i} & =\left\langle\psi_{e}\left|H_{i}(q)\right| \psi_{e}\right\rangle-\left\langle\psi_{g}\left|H_{i}(q)\right| \psi_{g}\right\rangle
\end{aligned}
$$

is introduced.

To go further in our analysis of the two-level model, we recall that the interaction between the subsystems can be presented as a sum of the resonance term and the Coulomb term:

$$
H_{i}(q)=H_{r}(q)+H_{c}(q) .
$$

The resonance term describes the one-electron hopping between the two subsystems. It can be written as

$$
H_{r}=-\sum_{i_{\sigma} j} b_{i j}(q)\left(r_{i \sigma}^{+} c_{j \sigma}+c_{j \sigma}^{+} r_{i \sigma}\right),
$$

where $b_{i j}(q)$ are the corresponding hopping (resonance) integrals; $r_{i \sigma}^{+}$and $c_{j \sigma}^{+}$are the operators creating an electron with the spin projection $\sigma$ on the $i$ th orbital of the RS and the $j$ th orbital of the $\mathrm{CS}$, respectively. The summation in Eq. (14) is extended to all the atomic orbitals in the respective subsystems.

The Coulomb term contains the repulsion between the electrons in the different subsystems and the attraction of the electrons in one subsystem to the nuclei of its counterpart. If we neglect the penetration effects for the Coulomb interaction 
between the two subsystems, the Coulomb operator can be written as follows:

$$
H_{c}=\sum_{i, j} g_{i j}(q)\left(\hat{n}_{i}-Z_{i}\right)\left(\hat{n}_{j}-Z_{j}\right),
$$

where $\hat{n}_{i}$ and $\hat{n}_{j}$ are the corresponding operators of the numbers of electrons and the quantities $g_{i j}(q)$ are the Coulomb interaction integrals for the $i$ th and $j$ th atoms in the respective subsystems; $Z_{i}$ and $Z_{j}$ are the respective core charges.

Since the basis functions $\psi_{g}$ and $\psi_{e}$ are built of the eigenfunctions of the subsystems, which have the certain number of electrons, the resonance operator does not contribute to the diagonal matrix element of the interaction operator:

$$
\begin{aligned}
\left\langle\psi_{g}\left|H_{i}\right| \psi_{g}\right\rangle & =\left\langle\psi_{g}\left|H_{c}\right| \psi_{g}\right\rangle, \\
\left\langle\psi_{e}\left|H_{i}\right| \psi_{e}\right\rangle & =\left\langle\psi_{e}\left|H_{c}\right| \psi_{e}\right\rangle .
\end{aligned}
$$

The quantity $\Delta E_{i}$ depends only on the redistribution of the charge between the parts of the supermolecule. To simplify our qualitative analysis, we assume that all the Coulomb integrals $g_{i j}$ have the same value $g_{M R}$ which approximately describes the interaction between the charges localized in the two subsystems. Then the Coulomb term can be rewritten as follows [19]:

$$
H_{c}=g_{M R}\left(\hat{N}_{R}-Z_{R}\right)\left(\hat{N}_{M}-Z_{M}\right),
$$

which is simply the interaction of the net charges residing in the subsystems. If the subsystems have the same net charges in the states $\psi_{g}$ and $\psi_{e}$, the Coulomb contributions to their diagonal energies are equal and $\Delta E_{i}$ vanishes. It happens when the ${ }^{2 S_{R}^{\prime}+1} \Gamma_{R}^{\prime}$ and ${ }^{2 S_{M}^{\prime}+1} \Gamma_{M}^{\prime}$ states of the RS and CS are the excited states of the respective subsystems rather than the ionized ones. By contrast, when the ${ }^{2 S_{R}^{\prime}+1} \Gamma_{R}^{\prime}$ and ${ }^{2 S_{M}^{\prime}+1} \Gamma_{M}^{\prime}$ states are obtained from the ${ }^{2 S_{R}+1} \Gamma_{R}$ and ${ }^{2 S_{M}+1} \Gamma_{M}$ states by the transfer of electrons from one subsystem to another, the quantity $\Delta E_{i}$ is important.

The formulas of the two-level approximation provide a useful tool for the qualitative analysis of the organometallic reactivity (see the next section). They allow one to understand how the reactivity patterns of simple organic molecules are modified under the influence of metal-containing fragments. For any reaction which is restricted in the ${ }^{2 S_{R}+1} \Gamma_{R}$ ground (unreactive) state of the free reactants [i.e., for that with the high potential barrier on the PES $\left.E_{R}(q)\right]$ we must find some relatively low energetic excited or ionized state ${ }^{2 S_{R}^{\prime}+1} \Gamma_{R}^{\prime}$ of the RS with the PES $E_{R}^{\prime}(q)$ which would have the low barrier or even the valley along the same path (reactive state). If such a state cannot be found, we must conclude that there is no way to perform the desired transformation even in the ligand sphere of any metalcontaining fragment. However, the desired state usually exists (see below). In that case the superposition of the reactive state (with the weight factor $\sin ^{2} \varphi$ ) and that of the unreactive one (with the weight factor $\cos ^{2} \varphi$ ) lead to barrier lowering, as compared to the barrier of the ground-state reaction of the free reactants. The barrier lowers substantially when the weight of the reactive state $0<\sin ^{2} \varphi(q)<1$ is significant along the whole reaction path. From the simple two-level formulas (10)-(12) one can derive the obvious conditions on the electronic structure of the CS and RS that must be satisfied to make the organometallic reaction more feasible than the restricted ground-state reaction of the free reactants. For instance, the energy gaps $\Delta E_{R}(q)$ and $\Delta E_{M}$ between the excited or ionized states and the ground states of both the CS and RS must be not very large as compared to the interaction matrix element $b$.

\section{Applications}

In this section we apply our general theory in its approximate two-level form to a series of examples. Our approach will have the same structure each time. We consider, first, a model PES (EP) for a class of chemical transformations and analyze the reasons why a barrier exists on the EP of the free reactants. Second, we find the excited or ionized state of the free RS which has either a low barrier or a valley along the same path. In the third step we construct a model for the interaction between the RS and CS and analyze the characteristics of the electronic structure of the cs which control its capacity to modify the EP of the unreactive state by means of its superposition with that of the reactive one.

Generally, none of the three cited steps of our analysis is trivial. In the examples which follow the ways to choose the basis states $\psi_{8}$ and $\psi_{e}$ of the corresponding two-level models (i.e., first two steps) are, probably, the most transparent. However, even in these relatively simple examples, it is not that easy to find the relevant interaction acting in the restricted two-dimensional space and ensur- 
ing effective superposition of the reactive state with the unreactive one. From time to time some exotic interactions (such as vibronic, spin-orbital, exchange, etc.) are used in the literature to explain the modification of the chemical behavior of coordinated species. However, we have not introduced any of these interactions in our operator $H_{i}$ [Eq. (13)] and restrict ourselves to the leading intersubsystem interactions: with resonance and Coulomb interactions. These interactions are in a sense fundamental. They are not introduced ad hoc but are present in the exact electronic Hamiltonian of the supermolecule. We shall show that the fundamental interactions responsible for the chemical bonding between the subsystems are also effective in the modification of their chemical behavior.

In some cases (see below) we encounter certain contradictions between the demand of using only the fundamental interactions and that of using only two basis states. For example, the resonance interaction can admix only the states which differ by one electron transferred from one subsystem to another. If the supermolecule in its two basis states $\psi_{g}$ and $\psi_{e}$ has the same net charges on the constituent subsystems, matrix element of the resonance interaction vanishes for those states. In this case we must replace the original resonance interaction by an effective one derived from the resonance to obtain the mixing of the basis states of sufficient strength. The meaning of the transition from the original interaction to the effective one is to take into account numerous intermediate states playing a subordinate role of the interaction mediators with electrons transferred from one subsystem to another. The effective interaction can be derived from the resonance interaction, for example, by the Löwdin partition technique $[17,19]$ or by some other method.

\section{REACTIONS CONTROLLED BY EXCHANGE REPULSION}

The potential energy surfaces of the overwhelming majority of reactions between molecules with closed electronic shells are controlled by the overlap and mixing of the orbitals of the reactants [20-22]. Let us consider a simple addition reaction:

$$
\mathrm{A}+\mathrm{B} \rightarrow \mathrm{A}-\mathrm{B} \text {. }
$$

Two reacting molecules $\mathrm{A}$ and $\mathrm{B}$ (or the product molecule $A-B$ ) compose the RS, and the nuclear coordinates $q$ describing the approach of the reac- tants to each other are the appropriate nuclear coordinates for the RS. The EP of the reaction of the free closed-shell reactants $A$ and $B$ can be treated according to [22].

According to $[22,23]$ the addition reaction has a barrier due to the exchange repulsion between the filled molecular orbitals of the reacting molecules. We assume here that one pair of the interacting orbitals (the orbitals $\varphi_{a}$ and $\varphi_{b}$ localized on the molecules A and B, respectively; $\varepsilon_{a}$ and $\varepsilon_{b}$ are the corresponding orbital energies) gives the main contribution to the total energy and controls the shape of the EP. The single determinant wave function for the ground state of the RS is the frozen core function $\Phi_{\text {core }}$ multiplied by four operators creating electrons with opposite spin projections on the orbitals $\varphi_{a}$ and $\varphi_{b}$ :

$$
\Phi_{R}^{0}(q)=r_{b \beta}^{+} r_{b \alpha}^{+} r_{a \beta}^{+} r_{a \alpha}^{+} \Phi_{\text {core }} .
$$

The EP of the RS has the form (see [22])

$$
E_{R}(q)=\text { const }+2\left[\left(\varepsilon_{a}+\varepsilon_{b}\right)-2 k_{a b}\right] S_{a b}^{2}(q) .
$$

The extended Hückel theory [24] uses $k_{a b}=$ $1.75\left(\varepsilon_{a}+\varepsilon_{b}\right) / 2$, so the square bracket is positive (see also [25]), and the EP of the RS contains a term increasing along the addition reaction path due to the increase of the overlap integral $S_{a b}(q)$.

To construct a reactive state for the RS, we remove one electron from it. It is easy to evaluate the EP $E_{R}^{\prime}(q)$ for the reactive $S_{R}^{\prime}=\frac{1}{2}$ state of the RS with one electron removed from the upper orbital $\varphi_{b}$ :

$$
E_{R}^{\prime}(q)=E_{R}(q)-\varepsilon_{b}-\Delta \varepsilon_{b a}(q),
$$

where $\Delta \varepsilon_{b a}(q)$ is given by [22]

$$
\Delta \varepsilon_{b a}(q)=\frac{\left(k_{a b}-\varepsilon_{b}\right)^{2} S_{a b}^{2}(q)}{\varepsilon_{b}-\varepsilon_{a}}>0 .
$$

The EP of the ionized RS contains a negative (stabilizing) contribution $-\Delta \varepsilon_{b a}(q)$ which is also proportional to the $S_{a b}^{2}(q)$. For that reason the EP $E_{R}^{\prime}(q)$ increases slower than $E_{R}(q)$ [see Fig. 1(a)].

Now let us consider possible effects of the cS on the EP of the addition reaction. Let us take the ground state of the isolated CS as a single Slater determinant as well:

$$
\Phi_{M}^{0}=\prod_{m} c_{m \beta}^{+} c_{m \alpha}^{+}|0\rangle,
$$

where the $c_{m \sigma}^{+}$are the operators creating an electron with the spin projection $\sigma$ on the $m$ th molec- 


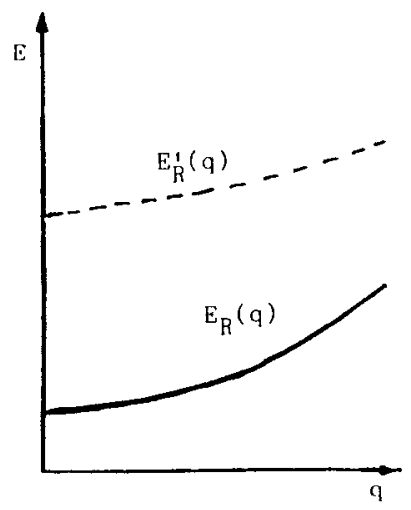

(a)

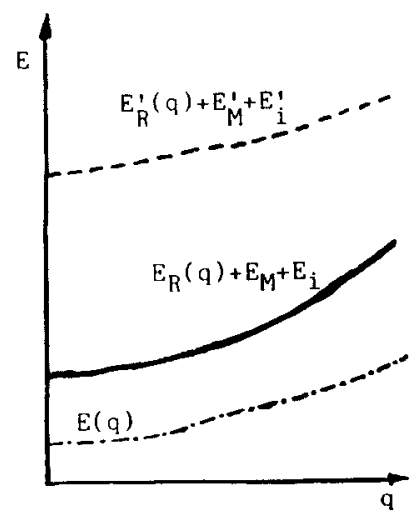

(b)

FIGURE 1. Model energy profiles for addition controlled by exchange repulsion. (a) Energy profiles for the free reactants. The energy profile for two filled orbitals coming together (the solid line), $E_{R}(q)$, increases rapidly due to the rapid increase of the overlap integral $S_{a b}(q)$ along the addition reaction path. The energy profile $E_{R}^{\prime}(q)$ is the same, but with one electron removed from the system (the broken line). This curve as a whole lies at higher energy since the extraction of an electron from Rs costs some energy, but the rate of increase is less along the reaction path since the coefficient at the $S_{a b}^{2}(q)$ in the geometry-dependent term in the expression for the energy is smaller. (b) Energy profiles for the coordinated reactants (supermolecule). Solid and broken lines are the same as in (a), but with the energies of the respective states of the $\operatorname{CS}\left(E_{M}\right.$ and $\left.E_{M}^{\prime}\right)$ and respective

intersubsystem interaction energies $\left(E_{i}\right.$ and $\left.E_{i}^{\prime}\right)$ added. They are also the diagonal energies of the basis states $\psi_{g}$ and $\psi_{e}$ [Eqs. (22), (23)]. Dotted line represents the supermolecule energy $E(q)$ obtained as a result of the quantum mechanical mixing of the basis states. The energy $E(q)$ increases slower than $E_{R}(q)$ but faster than $E_{R}^{\prime}(q)$. ular orbital of the CS. In our model problem the ground states of both the RS and CS are singlet. Therefore, the $\psi_{g}$ and $\psi_{e}$ states are singlets as well. The $\psi_{g}$ function is simply

$$
\psi_{g}=r_{b \beta}^{+} r_{b \alpha}^{+} r_{a \beta}^{+} r_{a \alpha}^{+} \Phi_{\text {core }} \wedge \Phi_{M}^{0} .
$$

The singlet function $\psi_{e}$, differing from $\psi_{g}$ by one electron transferred from the $\varphi_{b}$ orbital of the RS to an orbital $\phi_{v}$ which was empty in the ground state $\Phi_{M}^{0}$ of the CS, has the form

$$
\psi_{e}=2^{-1 / 2}\left(r_{b \alpha}^{+} c_{v \beta}^{+}-r_{b \beta}^{+} c_{v \alpha}^{+}\right) r_{a \beta}^{+} r_{a \alpha}^{+} \Phi_{\text {core }} \wedge \Phi_{M}^{0} .
$$

The EP for the supermolecule formed by the two ligands $\mathrm{A}$ and $\mathrm{B}$ and the metal-containing rest of the complex can be constructed now by making use of the two-level approximation [Eqs. (10)-(12)]:

$$
\begin{aligned}
E(q)= & {\left[E_{R}(q)+E_{M}+g_{M R} Q_{M} Q_{R}\right] } \\
& +\left[-\varepsilon_{b}-\Delta \varepsilon_{b a}-A_{M}\right. \\
& \left.+g_{M R}\left(Q_{M}-Q_{R}-1\right)\right] \\
& \times \sin ^{2} \varphi(q)+g \sin 2 \varphi(q),
\end{aligned}
$$

where

$$
\begin{gathered}
\tan 2 \varphi(q)=\frac{2 b}{\Delta E_{g e}}, \\
g=\left\langle\psi_{g}\left|H_{r}\right| \psi_{e}\right\rangle, \\
Q_{R}=-\left(N_{R}-Z_{R}\right), \quad Q_{M}=-\left(N_{M}-Z_{M}\right), \\
\Delta E_{g e}=-\varepsilon_{b}-\Delta \varepsilon_{b a}-A_{M}+g_{M R}\left(Q_{M}-Q_{R}-1\right) .
\end{gathered}
$$

Here, $A_{M}$ is the electron affinity of the metal-containing part of the supermolecule; $Q_{R}$ and $Q_{M}$ are the net charges of the RS and CS in the state described by the pure basis function $\psi_{g}$.

One may ask how it is possible to use a scheme with nonorthogonal orbitals for the reactants together with the Zero Differential Overlap (ZDO) formulas of the two-level approximation. We do not think it is a big problem. The nonorthogonality of the CS and RS orbitals will not produce any new effect and can be neglected for the purposes of the qualitative analysis we present here. By contrast the nonorthogonality is crucially important and must be explicitly included for the RS itself, since it is the physical origin of the exchange repulsion and of the barrier formation.

The EP for the supermolecule Eq. (24) is less repulsive than the EP of the addition of the free 
molecules A and B. The shape of the latter coincides with that of the first term of the EP of the supermolecule. However, the total EP Eq. (24) contains also two terms [those proportional to $\sin ^{2} \varphi(q)$ and $\sin 2 \varphi(q)$ ], both decreasing along the addition reaction path. The EPS discussed above are presented in Figure 1(b).

The above formulas probably explain the role of the metal complexes in a series of addition processes which do not occur for the free reagents but proceed more or less easily in the organometallic version. The metal serves here as a depository for an electron which otherwise would interfere the addition through the four-electron (exchange) repulsion. The situation thus resembles in a certain respect that of the general acid catalysis [26] where the protonated (electron-poor) form of a reagent is more reactive than the intact one. However, there is an important difference: In the case of the acid catalysis the electron-poor forms actually exist and in some situations can be chemically characterized as individual compounds, whereas in our case they are present only virtually, in the quantum mechanical sense, as contributions to the total wave function, and cannot be detected as individual particles.

Elsewhere $[25,27]$ the above theoretical scheme was applied to the analysis of the $\mathrm{CO}$ insertion into the metal-carbon bond:

$$
\mathrm{R}-\mathrm{ML}_{n}+\mathrm{CO} \longrightarrow \mathrm{R}-\underset{\mathrm{O}}{\mathrm{C}}-\mathrm{ML}_{n} .
$$

The $\sigma$-bonded alkyl or aryl ligands are formally described as coordinated anions, and the charge ascribed to the aryl or alkyl $\sigma$-ligand $\mathrm{R}$ by the formal electron counting rules is -1 , as it should be in the free anion.

The addition of free $\mathrm{R}^{-}$to free $\mathrm{CO}$ never takes place. However, when the unit $R$ serves as a $\sigma$ ligand in complexes of a large variety of transition (and not only transition [28]) metals, the CO insertion readily occurs and the coordinated product of $\mathrm{R}^{-}$addition to $\mathrm{CO}$ appears. The difference between the free $\mathrm{R}^{-}$anion and the coordinated moiety $R$ is precisely that in the latter case an electron is partially transferred to the rest of the complex. Within our method this is described by the admixture of the $\psi_{e}$ state to the $\psi_{g}$ state.

The details of the $\mathrm{CO}$ insertion reaction with use of the proposed theory have been analyzed in $[25,27]$. It has been shown that two key quantities controlling the capacity of $\mathrm{CO}$ to insert in the
$M-R$ bonds are the ionization potential of the free $\mathrm{R}^{-}$anion $I_{R}$ and the electron affinity of the metal-containing part of the complex $A_{M}$. It is easy to understand their importance from Eq. (24), since the energy denominator in Eq. (24) is estimated as

$$
\Delta E_{g e} \approx I_{R}-A_{M}+g_{M R}>0 .
$$

The larger is $A_{M}$ and the smaller is $I_{R}$, the larger is the weight of the reactive basis state $\psi_{e}$ in the ground state of the metal complex. Respectively, the insertion proceeds easier for larger $A_{M}$ and smaller $I_{R}$. In papers $[25,27]$ we compared the ionization potentials for a series of aryl anions $\mathrm{R}^{-}$ and the electron affinities for a series of the metal complexes $\mathrm{ML}_{n}^{+}$with the experimental data on the reactions of CO insertion in the complexes $R M L_{n}$. Almost in all cases we found a fair agreement between the experiment and our theory. The anions with low ionization potentials and the complexes with high electron affinities favor the $\mathrm{CO}$ insertion.

There is a large variety of other reactions formally described as insertions of electron-rich (unsaturated) molecules containing double or triple bonds into the metal-element $\sigma$-bonds $\mathrm{M}-\mathrm{Nu}$ (for a more detailed review and reference see [29]). Clearly, the reactions of that type are very unfavorable for the free unsaturated molecules if the nucleophile also appears as a free anion $\mathrm{Nu}^{-}$. (In chemical terms that would be an attack of a nucleophile to an electron-rich molecule.) At the same time, for a variety of metals, unsaturated molecules, and $\sigma$-bonded nucleophiles the reaction proceeds [29], giving corresponding insertion products. We think that in all cases when the modification of the reactivity of the $\mathrm{Nu}^{-}$anions toward unsaturated molecules through the complexation of the former to a metal complex takes place, it always occurs due to the virtual electron transfer from the $\mathrm{Nu}^{-}$lone pair to a virtual orbital of the metal-containing fragment. The ionization potential of the $\mathrm{Nu}^{-}$anion and the electron affinity of the metal complex fragment $\mathrm{ML}_{n}^{+}$control the insertion of unsaturated molecules in the $\mathrm{M}-\mathrm{Nu}$ $\sigma$-bonds. Although this result has been reported (see references in $[25,27])$, it has never been obtained as a result of a subsequent derivation.

We do not discuss here another possible approach to the modification of the reactivity by the coordination, namely, that concerned with the activation of unsaturated compounds toward the nu- 
cleophilic attack developed in [30]. It also can be reformulated in terms of the admixture of the reactive (excited/ionized) states to the ground state of the unsaturated molecule which is inactive with respect to the nucleophilic attack.

A very similar situation has been recently discussed in the context of interactions on the metal surfaces. Hoffmann and co-workers [31] considered the possibility of the modification of the interaction pattern of the rare gas atoms under the influence of the metal surface. Two rare-gas atoms repel each other due to the four-electron (exchange) repulsion of their completely filled electronic shells. Partial transfer of electrons from the filled electronic bands of the rare-gas monolayer to the empty bands of the metal surface was expected to result in some bonding between the adsorbed rare-gas atoms (i.e., in an attractive EP for the pair of atoms). It turned out, however, that the actual electron transfer from the rare-gas monolayer to the metal surface and the respective modification of the interatomic interaction were extremely weak. In our terms this result means that the admixture coefficients $C_{i k}^{0}$ of the states with electrons transferred from the RS (in this case the rare-gas monolayer) to the Cs (in this case them metal surface) are also very small, which results in the correspondingly weak modification of the interatomic interaction within the monolayer (for detailed discussion see [27]).

\section{REACTIONS RESTRICTED BY THE WOODWARD-HOFFMANN RULES}

Here we apply the general approach proposed in the theory section to a system with intersecting orbitals of different symmetry and consider the effect of its coordination to a transition metal complex (TMC) on the EP of the symmetryrestricted transformation [32]. We consider the symmetry-restricted isomerization reaction:

$$
\mathrm{A} \longrightarrow \mathrm{B}
$$

In the isomer A the molecular orbital $\varphi_{a}$ is occupied and $\varphi_{b}$ is empty, whereas in the isomer B the occupancies of the two are reversed and the occupancies of all other orbitals do not change. The symmetries of the orbitals $\varphi_{a}$ and $\varphi_{b}-\Gamma_{a}$ and $\Gamma_{b}$ are different. The intersection of the orbitals means that the orbital energies $\varepsilon_{a}$ and $\varepsilon_{b}$ become equal in some point $q^{\neq}$on the reaction path. In the vicinity of this point the energies can be approximated by the straight lines

$$
\varepsilon_{a}=k_{a}\left(q-q^{\neq}\right), \quad \varepsilon_{b}=-k_{b}\left(q-q^{\neq}\right)
$$

with $k_{a}$ and $k_{b}$ both positive numbers. The effect of all other electrons and nuclei is modeled by a harmonic potential $K\left(q-q_{0}\right)^{2} / 2$ which prevents the system from escaping to the infinite values of $q$.

To cover all the segments of the reaction path between the reactant and the product, the standard $3 \times 3$ configuration interaction (CI) expansion, (for reference see [9]) is used for the wave function of the RS. Four Slater determinants are involved:

$$
r_{a \beta}^{+} r_{a \alpha}^{+} \Phi_{\text {core }}, r_{b \beta}^{+} r_{b \alpha}^{+} \Phi_{\text {core }}, r_{b \alpha}^{+} r_{a \beta}^{+} \Phi_{\text {core }}, r_{b \beta}^{+} r_{a \alpha}^{+} \Phi_{\text {core }},
$$

where

$$
\Phi_{\text {core }}=\prod_{c} r_{c \beta}^{+} r_{c \alpha}^{+}|0\rangle
$$

and $c$ runs over the occupied orbitals of the molecular core.

The electronic Hamiltonian matrix in this basis falls into two $2 \times 2$ blocks: one corresponding to the $A_{1}$ symmetry,

$$
\begin{aligned}
& 2 \varepsilon_{a}+(a a \mid a a)+\frac{1}{2} K\left(q-q_{0}\right)^{2}, \quad(a b \mid b a), \\
& (a b \mid b a), \quad 2 \varepsilon_{b}+(b b \mid b b)+\frac{1}{2} K\left(q-q_{0}\right)^{2},
\end{aligned}
$$

and another to the $\Gamma_{a} \otimes \Gamma_{b}$ symmetry ( $\otimes$ stands for the tensor product of the two nondegenerate irreducible representations $\Gamma_{a}$ and $\Gamma_{b}$ ),

$$
\begin{aligned}
\varepsilon_{a}+ & \varepsilon_{b}+(a a \mid b b)+\frac{1}{2} K\left(q-q_{0}\right)^{2}, \quad-(a b \mid b a), \\
& -(a b \mid b a), \quad \varepsilon_{a}+\varepsilon_{b}+(a a \mid b b)+\frac{1}{2} K\left(q-q_{0}\right)^{2} .
\end{aligned}
$$

The EPS for the ground and the two lower excited terms are given by

$$
\begin{gathered}
E_{R}(q)=\left(k_{a}-k_{b}\right)\left(q-q^{\neq}\right)+\gamma+\frac{1}{2} K\left(q-q_{0}\right)^{2} \\
-\left(\left(k_{a}+k_{b}\right)^{2}\left(q-q^{\neq}\right)^{2}+(a b \mid b a)^{2}\right)^{1 / 2}, \quad(27) \\
E_{R}^{\prime}(q)=\left(k_{a}-k_{b}\right)\left(q-q^{\neq}\right)+\gamma+\frac{1}{2} K\left(q-q_{0}\right)^{2} \\
-(a b \mid b a),
\end{gathered}
$$

where we put $(a a \mid a a)=(b b \mid b b)=(a a \mid b b)=\gamma$. The qualitative picture of the RS terms is given in Figure 2(a). The ground-state EP obviously has a maximum near the intersection point $q^{\neq}$. The first 


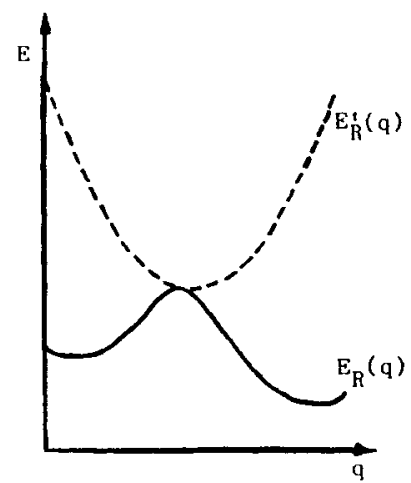

(a)

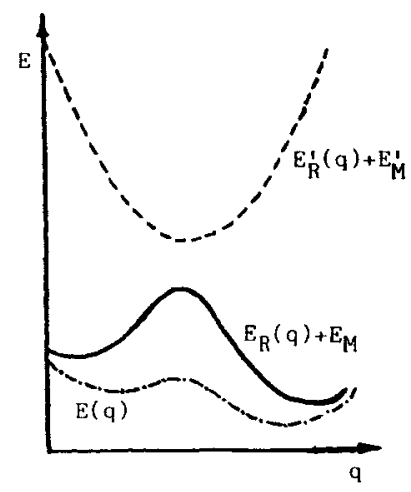

(b)

FIGURE 2. Model energy profiles for isomerization restricted by Woodward-Hoffmann rules. (a) Energy profiles for the free reactants. The energy profile for the ground-state isomerization, $E_{R}(q)$, has the high activation barrier (solid line). The energy profile $E_{R}^{\prime}(q)$ is of the same isomerization but in the triplet excited state, which has the valley (the broken line). (b) Energy profiles for the coordinated reactants (supermolecule). The solid and the broken lines are the same as in (a), but with the energies of the respective states of the CS $\left(E_{M}\right.$ and $\left.E_{M}^{\prime}\right)$ added. They are also the diagonal energies of the basis states $\psi_{g}$ and $\psi_{e}$, which have the same spin and symmetry [Eqs. (28), (29)]. The term $E(q)$ (dotted line) is the supermolecule energy obtained as a result of the quantum mechanical mixing of the basis states. The superposition results in a considerable reduction of the barrier as compared to the ground-state reaction of the free molecule $E_{R}(q)$.

excited term has the minimum approximately in the same position

The EP of the low-energy states obtained above fairly corresponds to the qualitative picture described elsewhere in this article as a prerequisite for the reactivity modification by the coordination. The reaction profile $E_{R}(q)$ has a barrier for the ground state ${ }^{2 S_{R}+1} \Gamma_{R}$ where $\Gamma_{R}=\mathrm{A}_{1}$ and $S_{R}=0$, whereas the energy profile $E_{R}^{\prime}(q)$ has a valley for the lowest excited state ${ }^{2 S_{R}^{\prime}+1} \Gamma_{R}^{\prime}$ with $S_{R}^{\prime}=1$ and $\Gamma_{R}^{\prime}=\Gamma_{a} \otimes \Gamma_{b}$.

It is also easy to find the metallic counterparts for the supermolecule basis states $\psi_{g}$ and $\psi_{e}$. As before the ground-state function $\left|\Gamma_{M} \gamma S_{M} \sigma\right\rangle$ of the metal complex without the isomerizing ligand $\mathrm{A}$ must be taken as the multiplier in $\psi_{g}$ :

$$
\psi_{g}=\left|\Gamma_{M} \gamma S_{M} \sigma\right\rangle \wedge\left|{ }^{1} \mathrm{~A}_{1}\right\rangle .
$$

For $\psi_{e}$ we have

$$
\begin{aligned}
\psi_{e}=\sum_{\gamma_{M}} \sum_{\sigma_{M}, \sigma_{R}}\left(\Gamma_{M} \gamma \mid \Gamma_{M}^{\prime} \gamma_{M}, \Gamma_{R}^{\prime}\right)\left(S_{M} \sigma \mid S_{M}^{\prime} \sigma_{m}, 1 \sigma_{R}\right) \\
\times\left|\Gamma_{M}^{\prime} \gamma_{M} S_{M}^{\prime} \sigma_{m}\right\rangle \wedge\left|\Gamma_{R}^{\prime} 1 \sigma_{R}\right\rangle
\end{aligned}
$$

The ${ }^{2 S_{M}^{\prime}+1} \Gamma_{M}^{\prime}$ excited state of the CS, having the lowest possible energy, must be used in the above expansion to make $\Delta E_{M}$ as small as possible.

Inserting the wave function (7), with $\psi_{g}$ and $\psi_{e}$ defined by Eqs. (28) and (29), into the general formulas of the two-level approximation and using the intersubsystem interaction given by Eqs. (14) and (15), we immediately find that the interaction parameter $b$ vanishes. The reason is quite evident: We did not include (as we did in the previous section) the states with an electron transferred between the subsystems. The resonance operator $H_{r}$ cannot admix the configurations with the same charge distribution, so no direct interaction is possible between the basis states $\psi_{g}$ and $\psi_{e}$ defined above. On the other hand, the distribution of electrons between the RS and CS does not change when the coordinated molecule $\mathrm{A}$ isomerizes to $\mathrm{B}$, and in that respect the choice of the basis states with the same net charges on the constituent subsystems seems to be reasonable. The charge transfer states merely provide the means for the interaction between the basis states of the constant number of electrons in the subsystems. The interaction can be treated in the framework of the effective Hamiltonian method (for reference see [19]). According to $[17,19]$ the original interaction operator $H_{r}$, having vanishing matrix elements within the subspace spanned by the wave functions $\psi_{g}$ and $\psi_{e}$, is replaced by the effective interaction operator $H_{r r}$ given by the formula

$$
H_{r r}=P H_{r} Q\left(E Q-Q H_{0} Q\right)^{-1} Q H_{r} P,
$$


where $P$ is the operator projecting the wave functions on the subspace spanned by the functions having the fixed number of electrons in each subsystem, $Q=1-P$, and

$$
H_{0}=H_{R}(q)+H_{M}+H_{c}
$$

The effective interaction operator $H_{r r}$ acts on the wave functions $\psi_{g}$ and $\psi_{e}$, but by the resolvent multiplier $\left(E Q-Q H_{0} Q\right)^{-1}$ it also takes into account all the states with electrons transferred between the two subsystems. The matrix element of the effective interaction operator does not vanish for the basis functions defined above:

$$
b=\left\langle\psi_{g}\left|H_{r r}\right| \psi_{e}\right\rangle \neq 0
$$

(an estimate for this quantity is given in the Appendix; see also [19]). Now, when $b \neq 0, \sin \varphi$ is nonvanishing as well. If its magnitude is significant, the barrier on the EP of the isomerization of $\mathrm{A}$ to $B$ in the ligand sphere of such a complex is strongly reduced as compared to that of the symmetry-restricted isomerization of the free molecule A. This reduction is due to the superposition of the EP of the unreactive ground state of A (B) with the $\mathrm{EP}$ of its reactive excited triplet state, and it takes place under the influence of the interaction with the CS. This is shown in Figure 2(b).

Many factors control the mixing parameter $\tan 2 \varphi$. The most obvious is the energy gap

$$
\Delta E_{M}=\left\langle\Gamma_{M}^{\prime} S_{M}^{\prime}\left|H_{M}\right| \Gamma_{M}^{\prime} S_{M}^{\prime}\right\rangle-\left\langle\Gamma_{M} S_{M}\left|H_{M}\right| \Gamma_{M} S_{M}\right\rangle .
$$

For the reaction to proceed easily, this gap must be as small as possible. For that reason the most probable candidates for the role of catalysts for the given type of reactions are transition metal complexes (TMCs) which normally offer a variety of low-energy (small $\Delta E_{M}$ ) excited states of their $d$ shell of different spin and symmetry.

It might seem to be not a great achievement since the abilities of TMCs as versatile catalysts are well known (see [1-4] and many other sources). However, the property of TMCs normally referred to as a crucial one for their catalytic activity is their ability to change their oxidation states easily. This property was really important in the previous section, where we discussed the modification of the reactivity in the processes restricted by the exchange repulsion and where the accessibility of the reduced state of the CS (i.e., its electron affinity) was one of the key factors. What is new in our characteristic of the TMCs as catalysts of the reactions restricted by the orbital symmetry (Woodward-Hoffmann) rules is that here their crucial property is the presence of the low-energy excited states of appropriate spin and symmetry. Namely their presence ensures the superposition of the unreactive ground state and the reactive triplet of the ligand undergoing the isomerization.

The above considerations can be supported by some experimental evidence. In [19] experimental data [33] on the catalytic activity of a series of metal porphyrins toward the isomerization of quadricyclane to norbornadiene have been analyzed. It turns out that the catalytic activity of the metal porphyrins fairly correlates with the presence of the low-energy $(d-d)$ states of appropriate total spin and symmetry in their spectra. In the case of the catalytically active $d^{7}$ and $d^{6}$ complexes of $\mathrm{Co}^{2+}, \mathrm{Co}^{3+}$, and $\mathrm{Fe}^{2+}$ the necessary states are present, whereas in the case of the catalytically inactive $d^{5}$ complexes of $\mathrm{Mn}^{2+}$ and $\mathrm{Fe}^{3+}$ the first $d-d$ excited states are separated from the respective ground states by a significant energy gap ( $2 \mathrm{eV}$ in the case of Mn porphyrin [34]). These data suggest that it is not the oxidation state of the transition metal ion that is relevant to its catalytic activity, but it is the number of $d$ electrons and the strength of the ligand field which together control the $d-d$ excitation spectrum [35]. This can probably explain some of the puzzling cases of the sharply contrasting catalytic activity of similar TMCS having the same ligands but different central ions. Although the quantum chemical parameters of transition metal ions change only slightly when passing from one metal to another, the $d-d$ spectrum may change drastically when the number of $d$ electrons changes.

The analysis presented in this section also proposes a general algorithm allowing us to find the reactive excited state which is to be admixed to the unreactive ground state. It turns out that the simple Pearson rule [36] guarantees the existence of the reactive excited state for any reaction restricted by the orbital symmetry rules in its ground state. The rule which describes the reactivity modification under the influence of light in the photochemical processes applies to the case of the catalytic reaction as well.

The theoretical model for the TMC catalysis of the symmetry-restricted reactions currently exist- 
ing in the literature was originally proposed in [37]. It is based on the direct application of the symmetry rules [32] to the TMC. It must be noted, however, that the single determinant approximation which is the conceptual basis for the Woodward-Hoffmann approach is not valid for the TMC where the electron correlations have crucial influence on the electronic structure of the molecules and on their transformations [19,38-40]. The Woodward-Hoffmann rules cannot be directly applied to the TMC. The inconsistency of the approach [37] in the particular case of the quadricyclane isomerization has been mentioned in [33]. Our approach proposed above goes beyond the single-determinant approximation and thus seems to be more appropriate for highly correlated electrons of TMC.

\section{REACTIONS FORBIDDEN BY THE SPIN CONSERVATION RULES}

The most important of the spin-forbidden processes [41] occurring in nature is oxidation with participation of the triplet dioxygen molecule. These reactions (similar to combustion) proceed at high temperatures by the free radical chain mechanism. On the other hand, numerous processes of the biochemical oxidation catalyzed by the TMC proceed smoothly with low activation energy, yielding highly specific products [42]. It is well established now (see [43] and references therein) that the active particles in a series of oxidation processes catalyzed by the metal porphyrins are the corresponding oxene complexes $\mathrm{O}=\mathrm{MP}$ $[\mathrm{M}=\mathrm{Fe}(\mathrm{III}), \mathrm{Mn}(\mathrm{II}) ; \mathrm{P}$ stands for a porphyrin-type macrocycle] irrespective of the source of oxygen atoms. These molecules act as one-oxygen donating reagents to a wide variety of organic substrates among which olefins and alkanes are most important. The RS relevant for the analysis of the reactivity of $\mathrm{O}=\mathrm{MP}$ complexes to organic substrates within the general framework of the second section of this article consists of the organic substrate molecule and the oxygen atom.

It is not generally realized that the addition of a free oxygen atom to organic substrates is a spinforbidden process as well. For large separations between the oxygen atom and the substrate molecule the ground state of the RS is triplet (the ground state of the oxygen atom is ${ }^{3} \mathrm{P}$ ) whereas the ground states of all the organic monoxygenation products (alcohols or epoxides) are singlet. Let us consider the reaction between the free reactants:

$\mathrm{O}(\mathrm{S}=1)+\operatorname{substrate}(\mathrm{S}=\mathrm{O})$

$$
\longrightarrow \text { substrate } \mathrm{O}(\mathrm{S}=\mathrm{O}) \text {. }
$$

Modification of the oxygen reactivity in this spinforbidden process must be described in terms of the superposition of the EPS of the triplet and the singlet states of the RS. For the reaction of the singlet oxygen atom (both in the ${ }^{1} \mathrm{D}$ and ${ }^{1} \mathrm{~S}$ states) with olefins and alkanes the EPs are available in the literature [44]. These reactions are very exothermic: The product minimum is $\sim 6 \mathrm{eV}$ lower than the separate reactants in the case of the alcohol formation from the alkane and the $O\left({ }^{1} D\right)$ atom. On this energy scale it seems reasonable to neglect the activation barrier of $\sim 2.5 \mathrm{kcal} / \mathrm{mol}$ [44]. The reaction of the $O\left({ }^{3} \mathrm{P}\right)$ atom yields different products. In [44] the EP is not given for the addition of the triplet $\left({ }^{3} \mathrm{P}\right)$ oxygen atom along the reaction path of the singlet atom addition, which is necessary for our analysis. It can however be easily sketched. The dissociation limit for the triplet term of an epoxide or alcohol molecule is $\sim 2 \mathrm{eV}$ (this is the ${ }^{3} \mathrm{P}-{ }^{1} \mathrm{D}$ energy separation of the oxygen atom) lower than that of the singlet term, or equivalently $\sim 4 \mathrm{eV}$ higher than the energy minimum of the corresponding singlet epoxide or alcohol. For the geometry corresponding to the singlet product minimum the energy of the triplet state can be estimated as the energy of the $\left(n \rightarrow \sigma^{*}\right)$ transition, which amounts to $\sim 7 \mathrm{eV}$ in the saturated alcohols and epoxides [45]. This means that for the product geometry the energy of the triplet state is $\sim 1 \mathrm{eV}$ higher than the dissociation limit of the singlet term and $\sim 3 \mathrm{eV}$ higher than the dissociation limit of the triplet state itself. Obviously, the energy of the triplet term increases along the path leading from the isolated oxygen atom and the substrate to the equilibrium geometry of the monoxygenation product. The singlet EP clearly decreases along this path. The qualitative picture of the EPS is given in Figure 3(a). It fairly corresponds to the Wigner definition of the spin-forbidden reaction [41] when the total spin of reagents, $S_{R}=1$, is not equal to that of the products, $S_{P}=0$.

Formally, the spin restriction in a chemical process is lifted in the presence of a paramagnetic TMC having the ${ }^{2 S_{M}+1} \Gamma_{M}$ ground state. An excited $2 S_{M}^{\prime}+1 \Gamma_{M}^{\prime}$ state of TMC is to be found such that the 


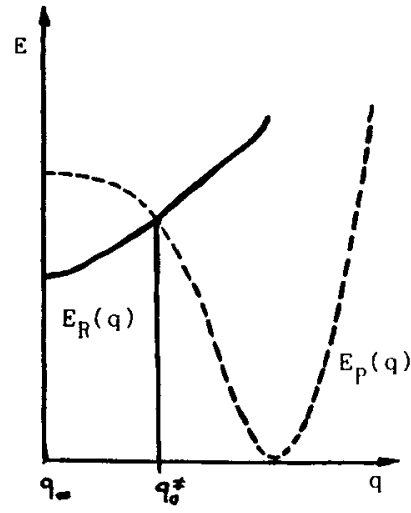

(a)

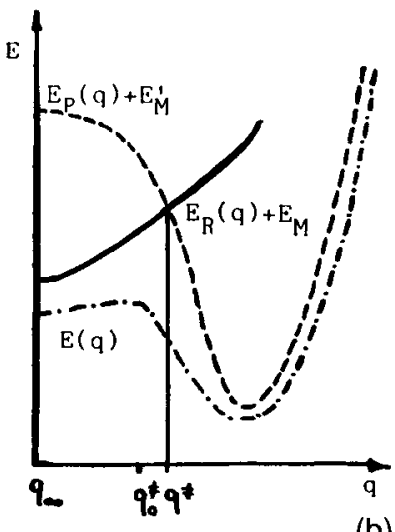

(b)

FIGURE 3. Model energy profiles for spin-forbidden monoxygenation. (a) Energy profiles for the free reactants and products. The energy profile $E_{R}(q)$ is for the addition of the free triplet oxygen atom to a singlet organic substrate molecule (solid line). This is the energy profile of the triplet reactants. The energy profile $E_{p}(q)$ for the addition of the singlet excited oxygen atom to the same substrate molecule (the broken line). This is the energy profile of the singlet monoxygenation product, which has the valley. Both profiles are taken along the same reaction path leading from the point $q_{x}$ corresponding to the infinitely separated oxygen atom and substrate molecule to the equilibrium geometry of the singlet monoxygenation product. The point $q_{0}^{\neq}$corresponds to the intersection of $E_{R}(q)$ and $E_{p}(q)$. (b) Energy profiles for coordinated reactants and products (supermolecule). Solid and broken lines are the same as in (a), but with the energies of the respective states of the $\operatorname{cs}\left(E_{M}\right.$ and $\left.E_{M}^{\prime}\right)$ added. They are also the diagonal energies of the basis states $\psi_{g}$ and $\psi_{e}$, which have the same total spin and the same point symmetry [Eqs. (34), (35)]. They intersect in the point $q^{\neq}$, which is further to the right from $q_{o}^{\neq}$. The term $E(q)$ (dotted line) is the supermolecule energy obtained as a result of the quantum mechanical mixing of the basis states. The mixing must be strong to ensure the considerable reduction of the activation barrier as compared to the bare activation energy, as shown. It is easy to see that there is a direct correlation between the electronic structure of the supermolecule and its EP. The greater the contribution of one of the basis states $\psi_{g}$ or $\psi_{e}$ to the ground state of the supermolecule, the closer the shape of the supermolecular EP to the EP of the corresponding state of the free RS.

spin selection rules

$$
\begin{aligned}
& S_{M}+S_{R} \geq S \geq\left|S_{M}-S_{R}\right| \\
& S_{M}^{\prime}+S_{P} \geq S \geq\left|S_{M}^{\prime}-S_{P}\right|
\end{aligned}
$$

and the point symmetry selection rules

$$
\Gamma_{M}^{\prime} \otimes \Gamma_{p}=\Gamma_{M} \otimes \Gamma_{R}=\Gamma
$$

are satisfied simultaneously. For paramagnetic TMC such states as a rule can be found. The electronic structure of the supermolecule is described here by the function of the form Eq. (7) with the basis states $\psi_{g}$ and $\psi_{e}$ defined by Eqs. (8) and (9) (with obvious substitution of $S_{P}$ and $\Gamma_{P}$ instead of $S_{R}^{\prime}$ and $\Gamma_{R}^{\prime}$ ) and with $\tan 2 \varphi$ determined by Eq. (10). For the electronic ${ }^{2 S+1} \Gamma$ state of the supermolecule comprising the reactants (products) and the TMC as a CS, the spin restrictions are formally lifted, which means that a single PES with the two minima corresponding respectively to the coordinated reactants and the coordinated products may exist. The PES for the transformation in the two-level approximation is given [with an obvious replacement of $E_{R}^{\prime}(q)$ by $\left.E_{p}(q)\right]$ by

$$
\begin{aligned}
E(q)= & \frac{1}{2}\left[E_{R}(q)+E_{M}+E_{P}(q)+E_{M}^{\prime}\right] \\
& -\frac{1}{2}\left\{\left[E_{R}(q)+E_{M}-E_{P}(q)-E_{M}^{\prime}\right]^{2}\right. \\
& \left.-4 g^{2}\right\}^{1 / 2}
\end{aligned}
$$

If the interaction matrix element $g$ is small, the ground state of the supermolecule is simply $\psi_{o}$ before the intersection point on Figure 3(b) and $\psi_{e}$ after it. The EP of the supermolecule coincides with $E_{R}(q)+E_{M}$ before the intersection point and with $E_{P}(q)+E_{M}^{\prime}$ after it.

Several definitions are to be introduced to discuss the structure of the model monoxygenation PES equation (32). Let $q_{\infty}(=0)$ correspond to the infinite separation between the reagents. We can also define the point $q_{0}^{\neq}$corresponding to the intersection of the free reagent and product term $\left[E_{R}\left(q_{o}^{\neq}\right)=E_{p}\left(q_{o}^{\neq}\right)\right]$and the energy of the free reagents and products at the point $q_{0}^{\neq}\left[\Delta E^{*}=\right.$ 
$\left.E_{R}\left(q_{0}^{\neq}\right)-E_{R}\left(q_{\infty}\right)\right]$. Another intersection point $q^{\neq}$ of the $E_{R}(q)+E_{M}$ and $E_{P}(q)+E_{M}^{\prime}$ curves [the solid and the broken lines in Fig. 3(b)],

$$
E_{R}\left(q^{\neq}\right)+E_{M}=E_{p}\left(q^{\neq}\right)+E_{M}^{\prime},
$$

or, alternatively,

$$
E_{R}\left(q^{\neq}\right)-E_{P}\left(q^{\neq}\right)=\Delta E_{M}=E_{M}^{\prime}-E_{M}
$$

allows one to define also the bare activation energy:

$$
\Delta E_{0}^{\neq}=E_{R}\left(q^{\neq}\right)-E_{R}\left(q_{\infty}\right) .
$$

The real activation energy for the process can be estimated as

$$
\Delta E^{\neq} \approx \Delta E_{0}^{\neq}-|g|,
$$

and the bare activation energy is itself a good estimate for the real activation energy if the interaction $|g|$ is small.

It is easy to see that there are two important quantities controlling the PES of the catalytic monoxygenation. These are the bare activation energy $\Delta E_{o}^{\neq}$and the interaction $|g|$. The bare activation energy $\Delta E_{o}^{*}$ is large. Indeed, consider the position of the intersection point $q^{\neq}$as a function of the excitation energy $\Delta E_{M}$ in the cs. At $\Delta E_{M}=$ $0, q^{\neq}=q_{0}^{\neq}$and $\Delta E_{0}^{*}=\Delta E^{*}$ (see above). The energy $\Delta E^{*}$ is high since it amounts to a considerable fraction of the excitation energy $E_{P}\left(q_{\infty}\right)$ $E_{R}\left(q_{x_{x}}\right)$ of the free RS. In the real Cs $\Delta E_{M}>0$ and we must have $q^{\neq}>q_{0}^{\neq}$to satisfy condition (33'). For the repulsive reagent term $E_{R}\left(q^{\neq}\right)>E_{R}\left(q_{o}^{\neq}\right)$, and respectively the bare activation energy $\Delta E_{o}^{\neq}$ is even higher than the energy $\Delta E^{*}$. The larger is the excitation energy $\Delta E_{M}$, the further we must go along the reaction path to satisfy condition (33'), the greater is the bare activation energy $\Delta E_{0}^{*}$. We can go even further and note that the bare activation energy is a quantity which is difficult to observe since it integrates the characteristics of both RS and CS. For that reason for a given process we replace the bare activation energy as a reactivity index by the excitation energy $\Delta E_{M}$ since they vary consistently: The bare activation energy $\Delta E_{0}^{\neq}$ increases with the excitation energy $\Delta E_{M}$.

For the small interaction $|g|$ the real activation energy is about $\Delta E_{0}^{*}$ and should be high (see above). By contrast, the experimental data show that the catalytic monoxygenation proceeds with low activation barriers (see $[42,43]$ and many other sources). This suggests that in the real monoxygenation processes the high $\Delta E_{o}^{*}$ is compensated by some strong interaction (i.e., $|g|$ is indeed large). Thus not only is the spin restriction formally lifted by coordination to a paramagnetic TMC but the high bare activation energy of the formally allowed reaction is compensated by interaction between the oxygen atom and the cs.

No reliable physical mechanism has ever been proposed for the interaction between the CS and RS which is strong enough $\left(|g| \approx \Delta E_{o}^{\neq}\right)$to account for the formation of the ground-state term with low activation barrier from the basis state $\psi_{g}$ and $\psi_{e}$ of the total ${ }^{2 S+1} \Gamma$ symmetry. To find an interaction between the RS and CS which is relevant to the problem of modification of reactivity in a spin-forbidden process, we consider the effect of the coordination of the oxygen atom on the metal porphyrin molecule. As a result of the coordination of the porphyrin, the ${ }^{3} \mathrm{P},{ }^{1} \mathrm{D}$, and ${ }^{1} \mathrm{~S}$ states of the oxygen atom [46] are split by the tetragonal field of the porphyrin molecule. Since the $2 p_{z}$ orbital of the oxygen points to the positively charged metal cation and has a strong stabilizing interaction with the empty $4 s$ orbital of the metal ion, its energy is reduced as compared to the energy of two remaining $p$ orbitals. The $2 p_{z}$ orbital is always occupied by two electrons. The $2 p_{x}$ and $2 p_{y}$ orbitals of the $e$ manifold home two remaining $p$ electrons of the oxygen atom. With this assumption we retain the ${ }^{3} \mathrm{~A}_{2}$ (the ground state), ${ }^{1} \mathrm{~B}_{2}$, and ${ }^{1} \mathrm{~B}_{1}$ states of the oxygen atom in the tetragonal field, being of the lower energy.

We consider the $d^{5} \mathrm{Mn}$ (II) and $\mathrm{Fe}(\mathrm{III})$ porphyrins. Their ground states are the ${ }^{6} \mathrm{~A}_{1}$ states of the $\left\{e^{2}\left({ }^{3} A_{2}\right) b_{2} a_{1} b_{1}\right\}$ configuration (the notation is with respect to the $C_{4 v}$ point group [47]). The total symmetry of the basis states $\psi_{g}$ and $\psi_{e}$ is given by the tensor product $A_{2} \otimes A_{1}=A_{2}$. To obtain the total $A_{2}$ symmetry for either the $B_{2}$ or $B_{1}$ states of the coordinated oxygen atom, we must use either the $B_{1}$ or $B_{2}$ states of the porphyrin, respectively. According to [47], the $d^{5}$ configuration permits only the quartet states of these symmetries. So the total spin of the states $\psi_{g}$ and $\psi_{e}$ is $\frac{3}{2}$ as well:

$$
\begin{aligned}
\psi_{g}= & \sum_{\sigma_{M}, \sigma_{R}}\left(3 / 2 \sigma \mid 5 / 2 \sigma_{M}, 1 \sigma_{R}\right)\left|A_{1} 5 / 2 \sigma_{M}\right\rangle \\
& \wedge\left|A_{2} 1 \sigma_{R}\right\rangle \\
& \psi_{e}=\left|\Gamma_{P} \otimes A_{2} 3 / 2 \sigma\right\rangle \wedge\left|\Gamma_{P} 0\right\rangle
\end{aligned}
$$

In these two basis states the number of electrons in the RS and CS corresponds to their number in the free reactants (neutral oxygen atom and substrate molecule) and in the free catalyst (metal por- 
phyrin). The number of electrons in the monoxygenation products is the same as it was in the reactants, so we can assume that the net charge transfer between the CS and RS vanishes, as in the case of the symmetry-restricted reactions (see the section on reactions restricted by the Woodward-Hoffmann rules). As in the case of the symmetry-restricted reactions the basis states $\psi_{g}$ and $\psi_{e}$ differ by excitations of electrons in the constituent subsystems. Using this analogy, we assume that the matrix element $g$ of the effective interaction between the states $\psi_{g}$ and $\psi_{e}$ appears due to the resonance one-electron transfers between the orbitals of the $e$ manifold of the transition metal ion and the $2 p_{x}$ and $2 p_{y}$ orbitals of the coordinated oxygen atom. We assume for the sake of definiteness that $\Gamma_{p}=B_{1}$. This case is covered by Eqs. (A5), (A8), and (A9) (see Appendix) with $x=(1 / 2)^{1 / 2}, \theta=-1, \beta(a)=\beta(b)=\beta$, and with the states $\psi_{g}$ and $\psi_{e}$ changing their roles. The resulting interaction matrix element $g$ is large enough to account for the compensation of the bare activation energy observed experimentally. The estimates of the analogous quantity given in [19] show that it amounts to about several electron-volts, which by far suffices to compensate the bare activation energy.

Now we return to the analysis of the properties of metal porphyrins controlling their activity in the monoxygenation. We found that the relevant excited state of the porphyrin is the ${ }^{4} B_{2}$ state of the $\left\{e^{2}\left({ }^{1} B_{1}\right) b_{2} a_{1} b_{1}\right\}$ configuration. Combining this result with Eqs. (10), (12), (32), we can see that the characteristic excitation energy of the CS controlling the bare activation energy and thus its activity in the reaction under consideration must be the energy $\Delta E_{M}=E\left({ }^{4} \mathrm{~B}_{2}\right)-E\left({ }^{6} \mathrm{~A}_{1}\right)$ of the metal porphyrin. The problem is that it is difficult to observe this energy directly. Nevertheless, we will try to analyze the experimental data on the reactivity of the oxygen atom coordinated to the metal porphyrin in terms of this quantity using our approach.

Elsewhere [48] it was found that there exists certain correlations between the catalytic activity of the substituted manganese (II) tetramesophenylporphyrines and the weight of the spin $\frac{5}{2}$ configuration in the isoelectronic Fe(III) complexes with the same macrocyclic ligands. The smaller the weight of the quartet in the Fe(III) complex, the greater the catalytic activity of the corresponding Mn(II) compound. According to [49], the weight of the quartet in the $d^{5}$ complexes is proportional to $(\zeta / \Delta)^{2}$, where $\zeta$ is the spin-orbital coupling constant and $\Delta$ is the energy separation between the ground ${ }^{6} \mathrm{~A}_{1}$ state of the metal porphyrin and its ${ }^{4} \mathrm{~A}_{2}$ state having the $\left\{\mathrm{e}^{2}\left({ }^{3} \mathrm{~A}_{2}\right) \mathrm{b}_{2}^{2} \mathrm{a}_{1}\right\}$ configuration. If we assume that the spin-orbital constant is not affected by the substitution, the unique reason for the change of the weight of the spin states is the variation of the energy difference $\Delta$. The catalytic activity of the metal porphyrin is controlled by the energy separation $\Delta E_{M}$ between the ground ${ }^{6} \mathrm{~A}_{1}$ state and the ${ }^{4} \mathrm{~B}_{2}$ state (see above). Since the substitution in the porphyrin ring affects the energies of these two states, it also affects both the reactivity of the oxygen atom coordinated to the $\mathrm{Mn}(\mathrm{II}) \mathrm{P}$ and the spin mixing in the isoelectronic Fe(III)P complex. To find the possible correlation, it is instructive to compare the data of [48] with the recent data on the catalytic activity of the octahalogenated porphyrin complexes of $\mathrm{Fe}$ (III) [50]. It turned out that the latter are more active than the unsubstituted compounds. The energy separations $\Delta$ and $\Delta E_{M}$ calculated by the method [40] change oppositely under the influence of the substitution: $\Delta$ increases, whereas $\Delta E_{M}$ decreases [51]. This behavior of $\Delta E_{M}$ agrees with the proposed role of the ${ }^{4} B_{2}\left\{e^{2}\left({ }^{1} B_{1}\right) b_{2} a_{1} b_{1}\right\}$ state. It seems to be reasonable to assume that the observed trends in the $\Delta$ and $\Delta E_{M}$ variations are universal. In all cases, when the substitution results in the increase of $\Delta$, it also causes the decrease of $\Delta E_{M}$ since both energies are controlled by the same crystal field parameters sensitive to the substitution. If this assumption is correct, it explains the experimental correlation [48] between the catalytic activity of the $\mathrm{Mn}(\mathrm{II})$ and the spin state of the isoelectronic Fe(III) porphyrins. The corresponding calculations are in progress now.

\section{Discussion}

Theoretical approaches which provide some general insight into the organometallic reactivity were developed mainly to describe the reactivity of adsorbed molecules [10-14], rather than the reactivity of the ligands, due to the obvious practical importance of the former. At the same time, from the fundamental point of view, that choice of the object for the theoretical studies cannot be considered the most successful. In the case of the surface-absorbed molecules we lack the information which is of crucial importance for any quantum chemical treatment. Thus any comparisons of 
predictions of the theoretical models of reactivity with experimental data are usually problematic because the real geometry of adsorbed molecule is not known for certain. In the case of the separate molecule of TMC we may hope to get information on its geometry, at least in principle. The same is correct for geometry estimates from quantum chemistry calculations. The geometry of separate molecules of TMC can be calculated at least in principle, but for a surface-adsorbed one this demands much more efforts. For these reasons in this article we concentrate on the analysis of the reactivity of coordinated molecules (ligands). The generalization of our approach to the case of adsorbed molecules is straightforward.

In our analysis of a complex problem of organometallic reactivity we turned to the basic principles of analysis of the complex systems adopted in quantum mechanics. After we divided the supermolecule undergoing the transformation into the reacting subsystem and the catalyst subsystem, our quantum chemical problem was reformulated in terms of the excited and/or ionized states of the isolated reactant molecules as well as those of the isolated TMC molecule. This approach not only reduced the original problem to a set of the simpler ones but also enabled us to construct a qualitative description of the organometallic reactivity and to discuss the latter in terms of the PESs of the excited/ionized states of the reagents.

For the qualitative analysis of the organometallic reactivity we proposed the two-level approximation, which seems to be successful for some important classes of reactions. It may well happen that in some cases the two-level approximation does not contain enough basis configurations to describe the modification of the reactivity. In this case the general equations must be used.

Although the theory presented in this article covers a wide class of organometallic reactions, it by no means exhausts the problem of the quantum mechanical treatment of the organometallic reactivity. When bond breaking and bond formation involve the metal atom, the reactions form another field of theoretical treatment and the quantities controlling this class of reactions may be different [6].

\section{Appendix}

Here we derive the approximate form of the effective interaction operator Eq. (30) acting in the subspace spanned by functions with a constant number of electrons in the CS and the RS. According to Eqs. (14) and (30),

$$
\begin{aligned}
H_{r r} & =P H_{r} Q R(E) Q H_{r} P, \\
R(E) & =\left(E Q-Q H_{0} Q\right)^{-1},
\end{aligned}
$$

where

$$
H_{r}=-\sum_{i_{\sigma} j} b_{i j}\left(r_{i \sigma}^{+} c_{j \sigma}+c_{j \sigma}^{+} r_{i \sigma}\right)
$$

The operator $P$ projects out all the states with electrons transferred from one subsystem to another. The operator $Q$ is complementary to $P$. The subspace spanned by $Q$ in its turn falls into two more subspaces $Q^{+}$and $Q^{-}$which correspond to an electron transferred to the CS and to the RS, respectively. We assume that all the states in each of these two subspaces have the same energy $\Delta E^{ \pm}$. Then the above expression can be rewritten as follows:

$$
\begin{array}{r}
H_{r r}=-P \sum_{i_{\sigma} j} \sum_{i_{\sigma^{\prime}}^{\prime}, j^{\prime}} b_{i j} b_{i^{\prime} j^{\prime}}\left(r_{i \sigma}^{+} c_{j \sigma} c_{j^{\prime} \sigma^{\prime}}^{+} r_{i^{\prime} \sigma^{\prime}}\left(\Delta E^{+}\right)^{-1}\right. \\
\left.+c_{j \sigma}^{+} r_{i \sigma} r_{i^{\prime} \sigma^{\prime}}^{+} c_{j^{\prime} \sigma^{\prime}}\left(\Delta E^{-}\right)^{-1}\right) P
\end{array}
$$

To proceed further, let us note that the catalyst orbitals involved in the above expressions must be of the same symmetry as the reactant ones to have the nonvanishing resonance integrals $b_{i j}$. Since we use the limited basis of the one-electron functions in the CS, we can assume that it contains only one orbital of each symmetry. In this case the resonance integrals become $b_{i j}=\delta_{i j} \beta(i), i, j=a, b$. Then, for the operator $H_{r r}$ the above formula takes the form

$$
\begin{aligned}
H_{r r}= & P \sum_{i, j} \sum_{\sigma, \sigma^{\prime}} \beta(i) \beta(j)\left(r_{i \sigma}^{+} r_{j \sigma^{\prime}} c_{j \sigma^{\prime}}^{+} c_{i \sigma}\left(\Delta E^{+}\right)^{-1}\right. \\
& \left.+r_{j \sigma^{\prime}}^{+} r_{i \sigma} c_{i \sigma}^{+} c_{j \sigma^{\prime}}\left(\Delta E^{-}\right)^{-1}\right) P \\
& +P \sum_{i, \sigma} \beta^{2}(i)\left[\left(\Delta E^{+}\right)^{-1} r_{i \sigma}^{+} r_{i \sigma}\right. \\
& \left.+\left(\Delta E^{-}\right)^{-1} c_{i \sigma}^{+} c_{i \sigma}\right] P .
\end{aligned}
$$

Substituting the functions $\psi_{g}$ and $\psi_{e}$ given by Eqs. 
(8) and (9), we get

$$
\begin{aligned}
b= & \left\langle\psi_{g}\left|H_{r r}\right| \psi_{e}\right\rangle \\
= & \sum_{i, j} \sum_{\tau, \tau^{\prime}} \beta(i) \beta(j) \\
& \times \sum_{\gamma_{M}, \gamma_{R}} \sum_{\sigma_{M}, \sigma_{R}} \sum_{\gamma_{M}^{\prime}, \gamma_{R}^{\prime}} \sum_{\sigma_{M}^{\prime}, \sigma_{R}^{\prime}}\left(\Gamma \gamma \mid \Gamma_{M} \gamma_{M}, \Gamma_{R} \gamma_{R}\right) \\
& \times\left(S \sigma \mid S_{M} \sigma_{M}, S_{R} \sigma_{R}\right)\left(\Gamma \gamma \mid \Gamma_{M}^{\prime} \gamma_{M}^{\prime}, \Gamma_{R}^{\prime} \gamma_{R}^{\prime}\right) \\
& \times\left(S \sigma \mid S_{M}^{\prime} \sigma_{M}^{\prime}, S_{R}^{\prime} \sigma_{R}^{\prime}\right) \\
& \times\left(\left(\Delta E^{+}\right)^{-1}\left\langle\Gamma_{R} \gamma_{R} S_{R} \sigma_{R}\left|r_{i \tau}^{+} \gamma_{j \tau},\right| \Gamma_{R}^{\prime} \gamma_{R}^{\prime} S_{R}^{\prime} \sigma_{R}^{\prime}\right\rangle\right. \\
& \times\left\langle\Gamma_{M} \gamma_{M} S_{M} \sigma_{M}\left|c_{j \tau}^{+}, c_{i \tau}\right| \Gamma_{M}^{\prime} \gamma_{M}^{\prime} S_{M}^{\prime} \sigma_{M}^{\prime}\right\rangle \\
& +\left(\Delta E^{-}\right)^{-1}\left\langle\Gamma_{R} \gamma_{R} S_{R} \sigma_{R}\left|r_{j \tau}^{+}, r_{i \tau}\right| \Gamma_{R}^{\prime} \gamma_{R}^{\prime} S_{R}^{\prime} \sigma_{R}^{\prime}\right\rangle \\
& \left.\times\left\langle\Gamma_{M} \gamma_{M} S_{M} \sigma_{M}\left|c_{i \tau}^{+} c_{j \tau},\right| \Gamma_{M}^{\prime} \gamma_{M}^{\prime} S_{M}^{\prime} \sigma_{M}^{\prime}\right\rangle\right) . \quad(\mathrm{A} 4)
\end{aligned}
$$

To find the matrix elements for some particular model of the Cs, we assume that the sufficient number of its electronic states can be described using two electrons placed in two orbitals of symmetries $\Gamma_{a}$ and $\Gamma_{b}$, respectively. Then four eigenstates are possible. They are two $\left|{ }^{1} \mathrm{~A}_{1}, \theta\right\rangle$ states,

$$
\begin{array}{r}
\left|\mathrm{cs}:{ }^{1} \mathrm{~A}_{1}, \theta\right\rangle=(1 / \sqrt{2})\left(c_{a \beta}^{+} c_{a \alpha}^{+}+\theta c_{b \beta}^{+} c_{b \alpha}^{+}\right)|0\rangle, \\
\theta= \pm 1,
\end{array}
$$

and the triplet and the singlet $\Gamma_{R}^{\prime}$ states,

$$
\begin{aligned}
\left|\mathrm{cs}:{ }^{1} \Gamma_{R}^{\prime}\right\rangle & =(1 / \sqrt{2})\left(c_{b \beta}^{+} c_{a \alpha}^{+}-c_{b \alpha}^{-} c_{a \beta}^{+}\right)|0\rangle, \\
\left|\mathrm{cs}:{ }^{3} \Gamma_{R}^{\prime}, 0\right\rangle & =(1 / \sqrt{2})\left(c_{b \beta}^{+} c_{a \alpha}^{+}+c_{b \alpha}^{+} c_{a \beta}^{+}\right)|0\rangle .
\end{aligned}
$$

The states $\psi_{g}$ and $\psi_{e}$ can be taken as follows:

$$
\begin{aligned}
\psi_{g}= & \left|\mathrm{RS}:{ }^{1} \mathrm{~A}_{1}\right\rangle \wedge\left|\mathrm{CS}:{ }^{1} \mathrm{~A}_{1}, \theta\right\rangle, \\
\psi_{e}= & \sum_{\sigma}(00 \mid 1 \sigma, 1-\sigma)\left|\mathrm{RS}:{ }^{3} \Gamma_{R}^{\prime}, \sigma\right\rangle \wedge \\
& \left|\mathrm{CS}:{ }^{3} \Gamma_{R}^{\prime},-\sigma\right\rangle .
\end{aligned}
$$

The ground state $\left|\mathrm{RS}:{ }^{1} \mathrm{~A}_{1}\right\rangle$ of the Rs is given by

$$
\left|\mathrm{RS}:{ }^{1} \mathrm{~A}_{1}\right\rangle=\left[x r_{a \beta}^{+} r_{a \alpha}^{+}-\left(1-x^{2}\right)^{1 / 2} r_{b \beta}^{+} r_{b \alpha}^{+}\right] \Phi_{\text {core }}
$$

where the positive number $x$ is determined by the diagonalization of the electronic Hamiltonian Eq. (26). Substituting all these wave functions into the above general expression, one finds

$$
\begin{aligned}
b=\left(\frac{3}{2}\right)^{1 / 2}[\theta x- & \left.\left(1-x^{2}\right)^{1 / 2}\right] \beta(a) \beta(b) \\
& \times\left[\left(\Delta E^{+}\right)^{-1}+\left(\Delta E^{-}\right)^{-1}\right] .
\end{aligned}
$$

For an alternative choice of the $\psi_{g}$ and $\psi_{e}$ states,

$$
\begin{gathered}
\psi_{g}=\left|\mathrm{RS}:{ }^{1} \mathrm{~A}_{1}\right\rangle \wedge\left|\mathrm{CS}:{ }^{3} \Gamma_{R}^{\prime}, 1\right\rangle, \\
\psi_{e}=\left|\mathrm{RS}:{ }^{3} \Gamma_{R}^{\prime}, 1\right\rangle \wedge\left|\mathrm{CS}:{ }^{1} A_{1}, \theta\right\rangle,
\end{gathered}
$$

the matrix element of the effective interaction operator becomes

$$
\begin{aligned}
b=(1 / \sqrt{2})\left[x-\theta\left(1-x^{2}\right)^{1 / 2}\right] \beta(a) \beta(b) \\
\times\left[\left(\Delta E^{+}\right)^{-1}+\left(\Delta E^{-}\right)^{-1}\right] .
\end{aligned}
$$

It is easy to see that the reactivity depends on the particular state of the cS used to construct either the $\psi_{g}$ or $\psi_{e}$ of the supermolecule. For $\theta=1$ the two terms in the brackets have the opposite sign and in the vicinity of the intersection point tend to cancel each other since there $x$ is $\sim 1 / \sqrt{2}$. On the other hand, the state with $\theta=-1$ tends to enhance the effective interaction, thus favoring the reactivity of the coordinated species.

\section{ACKVOWLEDGMENTS}

The author gratefully acknowledges the long years of collaboration with I. A. Misurkin and A. V. Soudackov. The author is also grateful to the referees for their comments.

\section{References}

1. C. Masters, Homogeneous Transition Metal Catalysis (Chapman \& Hall, London, 1981).

2. J. P. Candlin, K. A. Taylor and D. T. Thompson, Reactions of Transition Metal Complexes (Elsevier, Amsterdam, 1968).

3. Y. Yamamoto, Organotransition Metal Chemistry (Wiley, New York, 1986).

4. M. M. Jones, Ligand Reactivity and Catalysis (AP, New York, 1968).

5. M. J. Witko, Mol. Catal. 70, 227 (1991); N. Koga and K. Morokuma, Chem. Rev. 91, 823 (1991).

6. P. E. M. Siegbahn, J. Am. Chem. Soc. 115, 5803 (1993).

7. A. J. Shusterman, I. Tamir, and A. Pross, J. Organomet. Chem. 340, 203 (1988); A. Pross and S. S. Shaik, J. Am. Chem. Soc. 104, 187 (1982); S. S. Shaik and A. Pross, J. Am. Chem. Soc. (1982) 104, 5467; N. D. Epiotis, Theory of Organic Reactions (Springer-Verlag, Berlin, 1978).

8. C. C. Roothaan, J. Rev. Mod. Phys. 23, 69 (1951).

9. G. Naray-Szabo, P. R. Surjan and J. G. Angyan, Applied Quantum Chemistry (Reidel, Dordrecht, 1987). 
10. R. C. Baetzold, Adv. Catal. 25, 1 (1976).

11. R. A. van Santen, J. Chem. Soc. Faraday Trans. I 83, 1915 (1987).

12. R. A. van Santen, J. Mol. Struct. 173, 157 (1988).

13. A. B. Anderson, J. Mol. Catal. 54, 288 (1989).

14. E. Shustorovich, Adv. Catal. 37, 101 (1990).

15. W. Moffitt, Proc. Roy. Soc. A210, 245 (1951).

16. J. C. Tully, in Modern Theoretical Chemistry, Vol. 7, G. A. Segal, Ed. (Plenum Press, New York, 1977).

17. R. McWeeny and B. T. Sutcliff, Methods of Molecular Quantum Mechanics (Academic Press, New York, 1969).

18. J. S. Griffith, The Irreducible Tensor Method for Molecular Symmetry Groups (Prentice-Hall, London, 1962).

19. A. L. Tchougreeff and I. A. Misurkin, Dokl. Akad. Nauk SSSR 291, 1177 (1986) [in Russian]; A. L. Tchougreef and I. A. Misurkin, Theor. Eksp. Khim. 24, 398 (1988) [in Russian]; A. L. Tchougreeff and I. A. Misurkin, A. Chem. Phys. 133, 77 (1989).

20. K. Fukui, Theory of Orientation and Stereoselection (SpringerVerlag, Berlin, 1975).

21. R. Hoffmann, Acc. Chem. Res. 4, 71 (1971).

22. V. I. Minkin, B. Y. Simkin, and R. M. Minyaev, Quantum Chemistry of Organic Compounds (Springer-Verlag, Berlin, 1990).

23. R. Hoffmann, Solids and Surfaces: A Chemist's View of Bonding in Extended Structures (VCH, NY, 1988).

24. R. Hoffmann and W. N. Lipscomb, J. Chem. Phys. 36, 2179 (1962); R. Hoffmann and W. N. Lipscomb, J. Chem. Phys. (1962) 36, 3489; R. Hoffmann, J. Chem. Phys. (1963) 39, 1397.

25. A. L. Tchougreeff, Yu. V. Gulevich, I. A. Misurkin, and I. P. Beletskaya, Metallorganicheskaya Khimiya 1, 1159 (1988) [in Russian].

26. M. L. Bender, Mechanisms of Homogeneous Catalysis from Protons to Proteins. (Wiley, New York, 1971).

27. A. L. Tchougreef, Yu. V. Gulevich, I. A. Misurkin, and I. P. Beletskaya, J. Organomet. Chem. 455, 261 (1993).

28. D. G. Perez and N. S. Nudelman, J. Org. Chem. 53, 408 (1988).

29. J. P. Collman, L. S. Hegedus, J. R. Norton, and R. G. Finke, Principles and Applications of Organotransition Metal Chemistry (University Science Books, Mill Valley, CA, 1987).

30. O. Eisenstein and R. Hoffmann, J. Am. Chem. Soc. 102, 6148 (1980); Ibid. (1981) 103, 4308; Ibid. 103, 5582 (1981).
31. R. Hoffmann, M. Kersting, and Z. Nomicou, J. Chem. Phys. 95, 4033 (1991).

32. R. B. Woodward and R. Hoffmann, Conservation of Orbital Symmetry (Academic Press, New York, 1970); R. Hoffmann and R. B. Woodward, J. Am. Chem. Soc. 87, 2046 (1965).

33. J. Manassen, J. Catal. 18, 38 (1970); M. J. Chen and H. M. Feder, Ibid. 55, 105 (1978).

34. A. Harris, J. Chem. Soc. Faraday Trans. I 77, 369 (1981).

35. C. J. Ballhausen, Introduction to Ligand Field Theory (McGraw-Hill, New York, 1962)

36. R. G. Pearson, Symmetry Rules for Chemical Reactions (Wiley, New York, 1976).

37. F. D. Mango and J. H. Schachtschneider, J. Am. Chem. Soc. 89, 2484 (1967); F. D. Mango, Adv. Catal. 20291 (1969).

38. A. Veillard, Ed., Quantum Chemistry: The Challenge of the Transition Metals and Coordination Chemistry (D. Reidel, Dordrecht, 1986).

39. M. C. Böhm and R. Gleiter, Theor. Chim. Acta 59, 127 (1981); Ibid. 59, 153 (1981).

40. A. V. Soudackov, A. L. Tchougreeff, and I. A. Misurkin, Theor. Chim. Acta 83, 389 (1992).

41. E. Wigner, Nachr. Ges. Wiss. Güttingen 327 (1927); K. J. Laidler, The Chemical Kinetics of Excited States (Clarendon Press, Oxford, 1955).

42. K. V. Ingold, Acc. Chem. Res. 2, 1 (1969); L. L. Ingraham and D. L. Meyer, Biochemistry of Dioxygen (Plenum Press, New York, 1985).

43. B. Meunier, Chem. Rev. 92, 1411 (1992).

44. A. T. Pudzianowski and G. H. Loew, J. Am. Chem. Soc. 102, 5443 (1980); A. T. Pudzianowski and G. H. Loew, J. Mol. Catal. 17, 55 (1982).

45. A. J. Gordon and R. A. Ford, The Chemist's Companion (Wiley, New York, 1972).

46. E. U. Condon and G. H. Shortly, The Theory of Atomic Spectra. (Cambridge, 1953); C. E. Moore, Atomic Energy Levels (National Bureau of Standards, Washington, DC, 1949).

47. J. Otsuka, J. Phys. Soc. Japan 21, 696 (1966).

48. A. L. Tchougreeff, A. B. Solovyova, A. I. Samokhvalova, and I. A. Misurkin, Teor. i Eksp. Khim. 23, 428 (1987) [in Russian]; Theor. Exp. Chem. 23, 400 (1987) [in English].

49. M. M. Maltempo, J. Chem. Phys. 61, 2540 (1974).

50. J. F. Bartoli, O. Brigaud, P. Battioni, and D. Mansuy, J. Chem. Soc. Chem. Commun., 440 (1991).

51. A. V. Soudackov, private communication. 